\title{
Influence of water stress and exogenous glycinebetaine on sunflower achene weight and oil percentage
}

\author{
${ }^{1} N$. Iqbal, ${ }^{2 *} M$. Y. Ashraf and ${ }^{2} M$. Ashraf \\ ${ }^{1}$ Department of Botany University of Agriculture, Faisalabad, Pakistan \\ ${ }^{2}$ Nuclear Institute for Agriculture and Biology (NIAB), Faisalabad, Pakistan
}

\begin{abstract}
The study was carried out to assess whether exogenously applied glycinebtaine has any role in reducing the adverse effects of water stress on sunflower achene yield and oil contents. Two sunflower lines, Gulshan-98 and Suncross were subjected to water stress at the vegetative and reproductive stages of plant growth. Three levels of glycinebtaine (0, 50 and $100 \mathrm{mM}$ ) were applied before sowing (seed treatment) and at the time of initiation of stress at the vegetative and reproductive stages. A marked adverse effect of water stress on 100 -achene weight and achene oil contents were observed in both sunflower lines. Exogenous supply of glycinebtaine was not effective in alleviating the adverse effects of water stress on achene oil percentage. Foliar spray of glycinebtaine, however, significantly reduced the negative effects of water stress on achene weight. Seed treatment with either level of glycinebtaine was not effective in increasing the 100- achene weight and achene oil percentage under both normally irrigated and water stress conditions. The sunflower line, Suncross produced higher oil yield than that of Gulshan-98.
\end{abstract}

Key words: Drought, sunflower, glycinebtaine, yield, oil percentage

*Corresponding Author, E-mail: niabmyashraf@yahoo.co.uk

\section{Introduction}

The growth, development and spatial distribution of plants are severely restricted by a variety of environmental stresses. Among different problems faced by crop plants, water stress is considered to be the most critical one (Boyer, 1982). Plants being immobile cannot evade water stress in the same way as mobile organisms. So, they show many morphological and physiological alterations to acclimatize to unfavorable environment (Sakamoto and Murata, 2002). One such mechanism that is ubiquitous in plants is the accumulation of certain organic metabolites of low molecular weight that are collectively known as compatible solutes (Bohnert, et al., 1995). These compatible solutes are uniformly neutral with respect to the perturbation of cellular functions, even when present at high concentrations (Yancey, et al., 1982). Compatible osmolytes include sugars, proline and quaternary ammonium compounds. Glycinebtaine,an important quaternary ammonium compound is considered to be one of the most predominant and most effective osmoprotectant (Burnet, et al., 1995). It is commonly recognized as the only solute accumulated in higher plants submitted to osmotic stress, which satisfies all other solute requirements (Gorham, 1992). However, not all plants can produce osmolytes in sufficient quantities to combat drought. In crops with poor or no osmolytes accumulating ability, genetic engineering is a way to increase stress tolerance. To date this has had some success, but transgenic plants having compatible osmolytes genes show low level of synthesis of these solutes than required under stressful conditions (Ishitani, et al., 1995; Nolte, et al., 1997). As the biosynthesis of glycinebtaine is energetically costly (Hanson and Wyse, 1982) and most plants do not normally accumulate sufficient amount of osmolytes, the exogenous application of glycinebtaine has been suggested as an alternative approach to improve crop productivity under water stress (Makela, et al., 1996). This approach has got considerable attention from the researchers from last few years. Significant advances have been made in alleviating the effects of environmental stresses by exogenously applied glycinebtaine in different crops, such as tomato (Makela, et al., 1998), wheat (Allard, et al., 1998), and rice (Rahman, et al., 2002). Application of glycinebtaine also improves germination and seedling growth of many crop plants under stressful environment (Rukiye and Sebnem, 1993 and Gadallah, 1999). Sunflower (Helianthus annuus L.) is an important oilseed crop grown in different parts of the world. It has C3 photosynthetic 
pathway and is mostly cultivated in arid and semiarid regions. Although many studies on drought tolerance of sunflower has been carried out (Luisa, et al., 1995; Ashraf and O’Leary, 1996), basic research on role of exogenous glycinebtaine in drought tolerance of sunflower is scarce. The present study were conducted to find the responses of sunflower to water stress and to examine whether and how exogenous glycinebtaine ameliorates the effects of drought on sunflower achene weight and oil contents. This study has been done in Nuclear Institute for Agriculture and Biology (NIAB) on 2002.

\section{Materials and Methods}

Seeds of sunflower genotypes, Suncross and Gulshan-98 were obtained from the regional office of Pakistan Seed Council, Faisalabad. The study was conducted at the experimental area of Deptartment of Botany, University of Agriculture, Faisalabad, Pakistan. Water stress was applied at the vegetative and reproductive stages of plant growth. There were three treatments of glycinebtaine $(0,50$ and $100 \mathrm{mM}$.) which were applied before sowing (seed treatment), at vegetative stage and reproductive stage. The experiment was laid out in a split plot design with 8 replications for each experimental unit.

The plants were harvested at maturity and 100achene weight was recorded. Dried seeds (100 g.) of each experimental unit were crushed and fed to a Soxhlet extractor fitted with one liter round bottom flask and a condenser. The extraction was executed with $0.5 \mathrm{~L}$ of $\mathrm{n}$-hexane on a water bath for $6-7 \mathrm{~h}$. The solvent was distilled off under vacuum in a rotary evaporator and percentage of oil was recorded. Analysis of variance of the data from each attribute was computed using the MSTAT Computer Program (MSTAT Development Team, 1989). The Duncan's New Multiple Range test at 5\% level of probability was used to test the differences among mean values (Steel and Torrie, 1980).

\section{Results}

Highly significant $(\mathrm{P}>0.001)$ differences were observed among water deficit treatments with respect to achene weight (Figure 1). Imposition of water deficit at the vegetative stage caused greater reduction (37\%) in hundred-achene weight of stressed plants in respect of normally irrigated ones than that of the reproductive stage (24\%). The adverse effects of water deficit on hundred-achene weight were significantly reversed $(\mathrm{P}>0.005)$ by the exogenous application of glycinebtaine (GB) at different growth stages (Figure 3a). Foliar application of GB at the vegetative or thereprodu-ctive stage was proved to be more beneficial regarding hundredachene weight than that when GB was applied as seed treatment. The three levels of GB also produced significant $(\mathrm{P}>0.001)$ effects on hundred-achene weight (Figure 3b). Maximum hundred achene weight ( 4.5 g.) was recorded under the application of $50 \mathrm{mM} \mathrm{GB}$ followed by $100 \mathrm{mM}$. (4.4 g.) and 0 mM. (4.3 g.). The two sunflower lines differ significantly $(\mathrm{P}>0.001)$ with respect to hundredachene weight. The sunflower line, Gulshan-98 produced greater hundred-achene weight (4.5 g.) than that of Suncross (4.2 g.). Interactions among different factors such as water deficit treatment $x$ time of GB application and water deficit treatments $\mathrm{x}$ sunflower liens were statistically significant. Exogenous application of GB either in the form of seed treatment or foliar spray had no effect on hundred achene weight under normally irrigated conditions (Figure 1). When water deficit was imposed at the vegetative stage, exogenous application of GB was much effective when it was applied at the time of initiation of stress than that of seed treatment or foliar application of GB at the reproductive stage. In contrast, when water deficit was imposed at the reproductive stage, foliar spray of GB before the application of water stress (at the vegetative stage) and at the time of the initiation of stress (at the reproductive stage) have almost similar value (4.0 and $4.1 \mathrm{~g}$. respectively) for hundred achene weight which were significantly higher than that of plants raised from GB-treated seed. Achene oil percentage was determined only in plants showed some response to GB treatments at different growth stages. Since, seed treatment with GB showed non significant results in preventing the adverse effects of water deficit on yield and yield components of both sunflower lines hence it was excluded in determining achene oil contents. Plants exposed to water deficit at different growth stages showed significantly $(\mathrm{P}>0.001)$ low achene oil contents than that of normally irrigated ones (Figure 2). Imposition of water deficit at the vegetative and reproductive growth stages caused a 5.8, 5.6\% reduction in oil yield of water stressed plants as compared with their respective controls. Foliar application of GB at both the vegetative and reproductive growth stages was not effective in ameliorating the adverse effects of water deficit on achene oil percentage. The three levels of GB had almost similar effects on this 


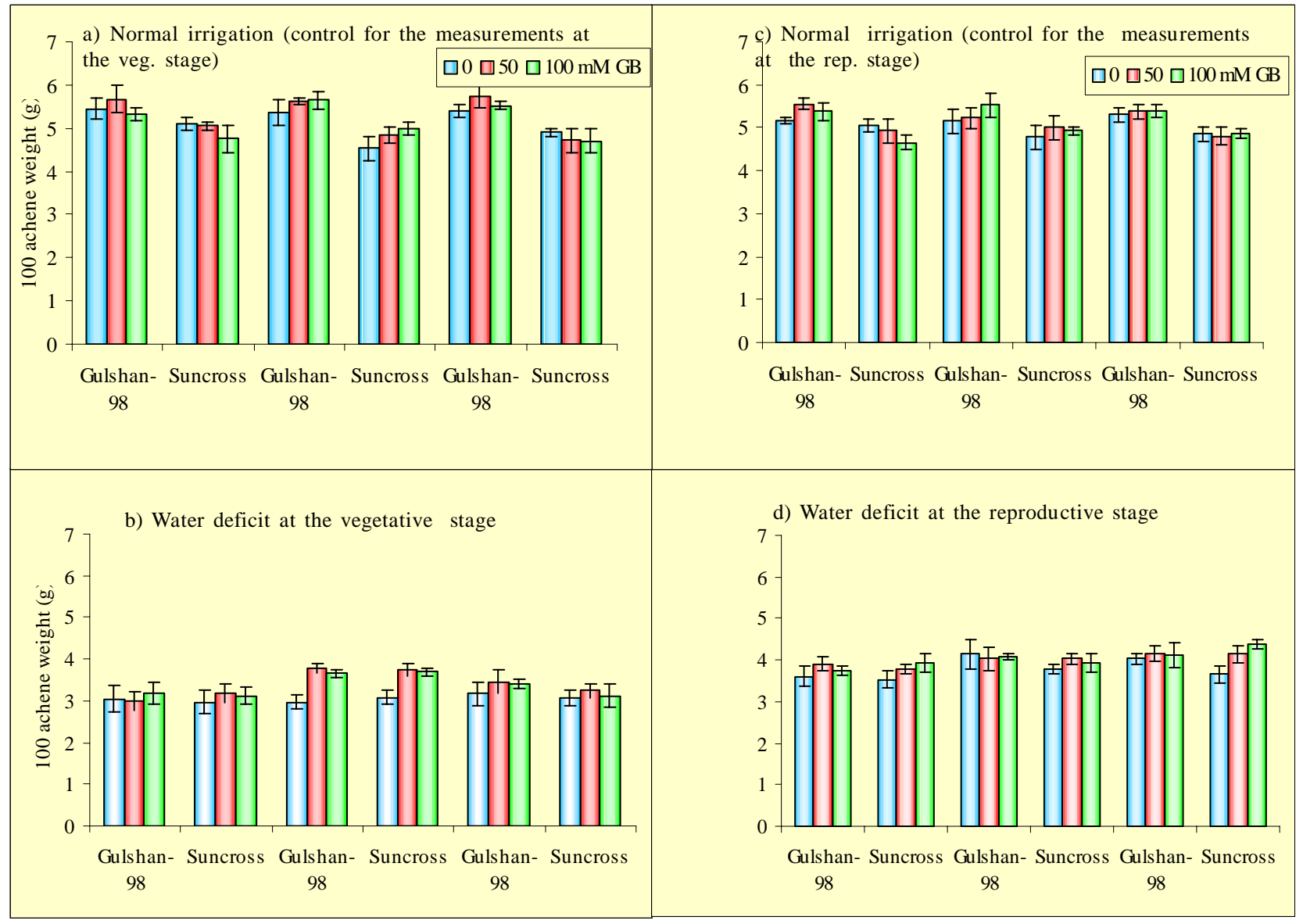

Figure 1: 100 achene weight (g) in two sunflower lines subjected to water deficit and glycinebetaine application at different growth stages

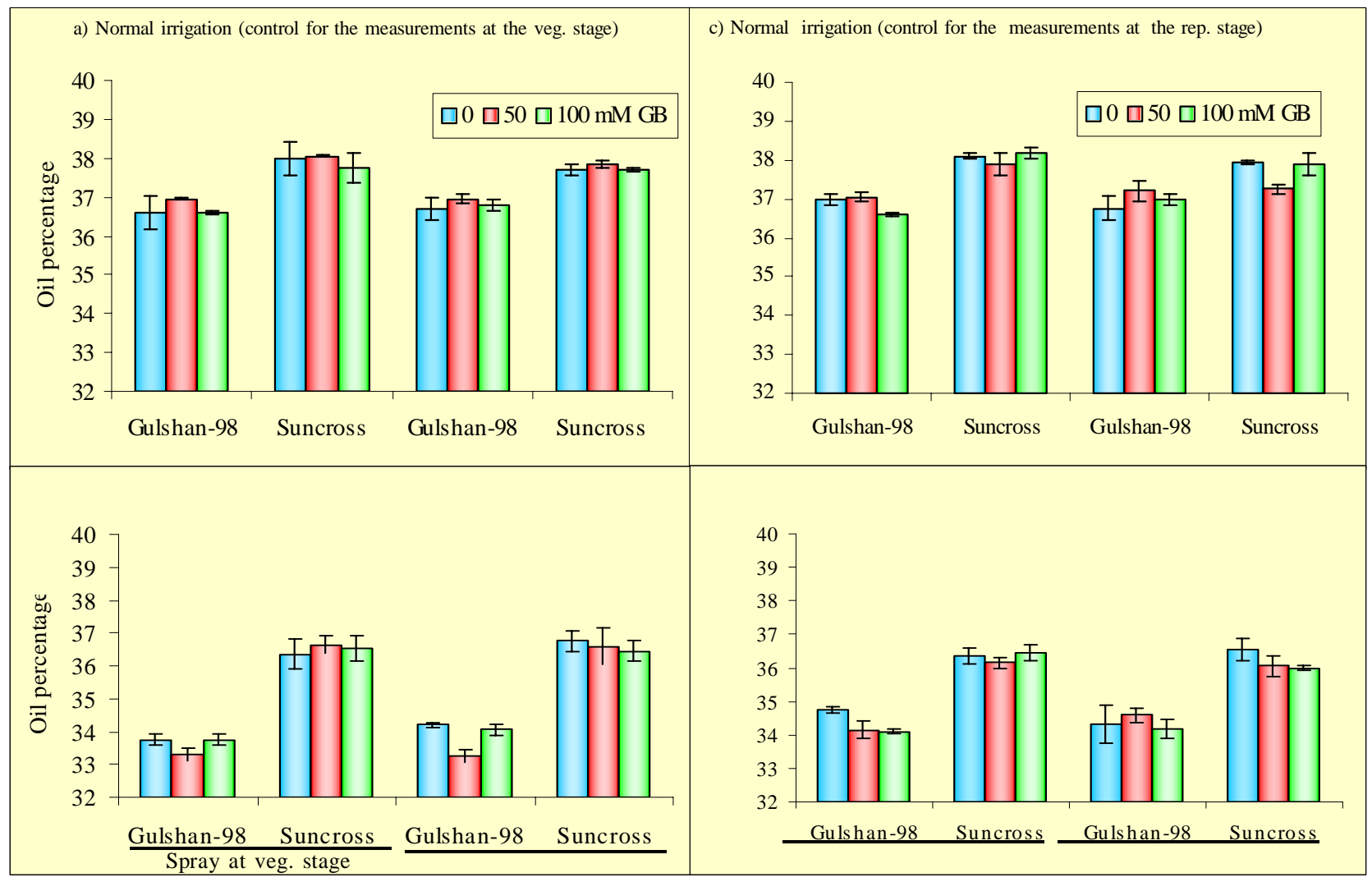

Figure 2: Oil percentage in two sunflower lines subjected to water deficit and glycinebetaine application at different growth stages 
variable. Interactions among water deficit treatments $\mathrm{x}$ time of GB application, water deficit treatments $\mathrm{x}$ GB levels and time of GB application x GB levels were also statistically non significant.

The sunflower lines exhibited highly significant ( $\mathrm{P}>0.001)$ differences for this variable. Suncross had higher achene oil contents (37.14\%) as compared with Gulshan-98 (35.44\%). Interactions among water $(\mathrm{P}>0.001)$. Under water deficit at the vegetative and reproductive stage, Suncross showed a 3 and $4 \%$ decrease in achene oil contents, respectively in water stressed plants as compared with unstressed ones. In contrast, a 8 and 7\% reduction in achene oil contents of Gulsha-98 was observed due to imposition of water deficit at the vegetative and reproductive stages respectively as compared with their controls.

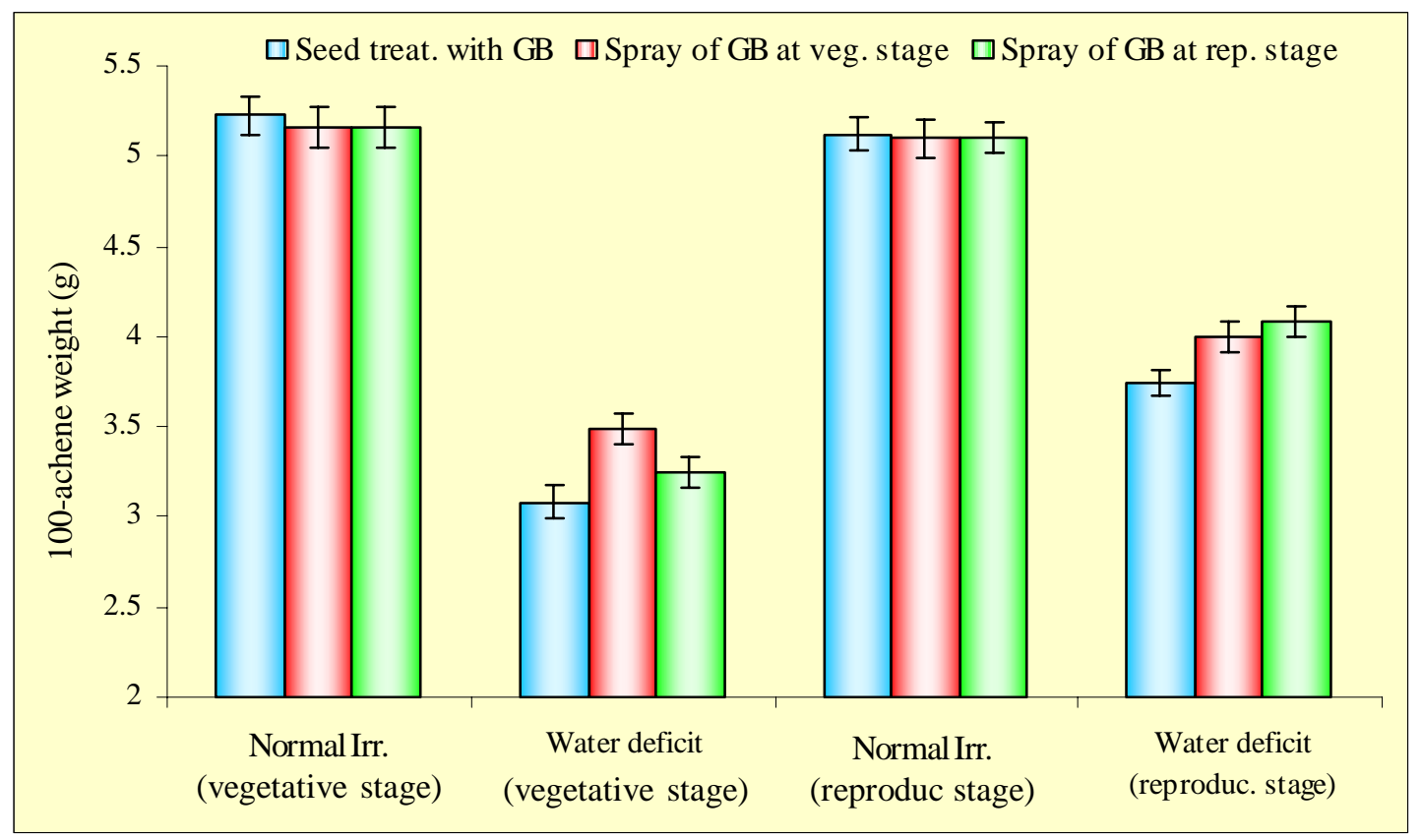

Figure 3a: Cumulative effect of exogenous GB application at different growth stages on 100 achene weight of sunflower lines under different water deficit treatments (time of GB x water deficit)

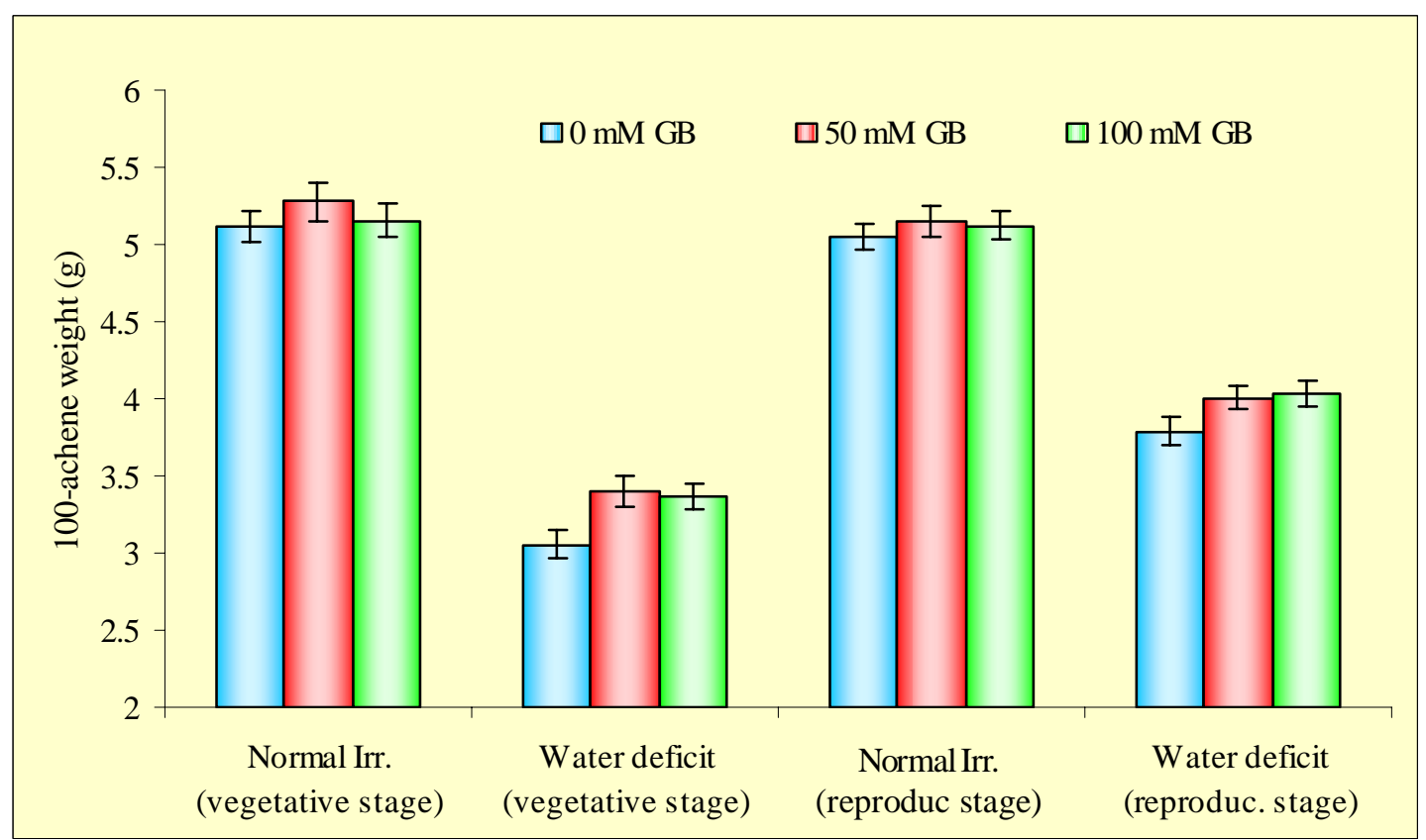

Figure 3b: Effect of different levels of GB on stomatal conductance of sunflower lines under different water deficit treatments (GB levels x water deficit) 


\section{Discussion and Conclusion}

This is a well established fact that yield of crop plants in drying soil reduces even in tolerant lines of that crop species (Ashraf and Mehmood, 1996; Tahir and Mehdi, 2002). A similar trend in yield decline was observed during the present investigation, the yield per plant and yield components were reduced due to water stress treatments. The decrease in yield and yield components in different sunflower genotypes has also been reported by many workers (Nandhagobal, et al., 1996; Prabhudeva, et al., 1998; Tahir and Mehdi, 2001; Tahir, et al., 2002). These workers clearly indicated that sunflower drought tolerant lines showed less reduction in yield plants in respect of susceptible lines. Hence, maintainenance of better yield of the sunflower line, Suncross than that of Gulshan-98 under water deficit environment as observed in the present study points towards its higher drought tolerance ability. The results of present experiment clearly indicated that pre-soaking of seeds with GB was not significant in alleviating the adverse effects of water deficit on achene yield of both sunflower lines. In contrast, Naidu, et al. (1998) reported a highly significant role of supplied GB in increasing the yield of cotton. However, the positive effects of foliar spray of GB 100- achene weight of sunflower lines grown under water limited environment as observed in the present study has also been however also reported in different crops such as tomato (Makela, et al., 1998), tobacco (Agboma, et al., 1997b), maize (Agboma, et al., 1997a), cotton (Gorham, et al., 2000) and wheat (Diaz-Zarita, et al., 2001). There are however, some contrasting reports indicating no effect of supplied GB on yield of wheat (Agboma, et al., 1997a) and cotton (Meek, et al., 2003).

It is clear from the results of the experiment that foliar application of glycinebtaine ameliorated the negative effects of water stress on achene biomass production in both sunflower lines. Achene oil percentage, however, was not affected by exogenous supply of glycinebtaine under both normally irrigated and water stress.

\section{Acknowledgement}

Authors are highly thankful to Dr. Sarwar Alam, Principal Scientist, NIAB, Faisalabad, Pakistan for reviewing this manuscript.

\section{References}

Agboma, P., M. G. K. Jones, P. Petonen-Sainio, H. Rita and E. pehu, Exogenous glycinebetaine enhances grain yield of maize, sorghum and wheat grown under two supplementary watering regimes. J. Agron. Crop Sci., (178): 29-37, 1997a

Agboma, P., P. Peltonen-Sanio, R. Hinkkanen and E. Pehu, Effect of foliar application of glycine betaine on yield components of drought stressed tobacco plants. Exp. Agri., 33:345-352, 1997b

Ashraf, M. and S. Mehmood, Response of four Brassica species to drought stress. Environ. Expt. Bot., 30: 93100, 1996

Ashraf, M. and J. W. O'Leary, Effect of drought stress on growth, water relations and gas exchange of two lines of sunflower differing in degree of salt tolerance. Int. J. Plant Sci., 157:729-732, 1996

Bohnert, H. J., D. E. Nelson and R. E. Jensen, Adaptation to environmental stresses. Plant Cell., 7:1099-1111, 1995

Boyer, J. S., Plant productivity and environment. Science., 218:443-448, 1982

Burnet, M., P. J. Lafontaine and A. D. Hanson, Assay, purification and partial characterization of Choline monoxygenase from spinach. Plant Physiol., 168:581588, 1995

Diaz-Zorita, M., M. V. Fernandez-Canigia and G.A. Grosso, Application of foliar fertilizers containing glycine betaine improve wheat yields. J. Agron. and Crop Sci., 186:209-215, 2001

Gadallah, M. M. A., Effects of proline and glycine betaine on Vicia faba responses to salt stress. Biol. Plant., 42:249-257, 1999

Gorham, J., Salt tolerance of plants. Science Progress., 76:273-285, 1992

Gorham, J., K. Jokinen, M. N. A. Malik and I. A. Khan, Glycinebetaine treatment improves cotton yields in field trails in Pakistan. Proc. World Cotton Res. ConfII, Athens, Greece, 624-627, 2000

Hanson, A. D., and R. Wyse, Biosynthesis, translocation and accumulation of betaine in sugar beet and its proginators in relation to salinity. Plant Physiol., 62:305-312, 1982

Ishitani, M., T. Nakamura, S. Y. Han and T. Takabe, Expression of betaine aldehyde dehydrogenase gene in barley in response to osmotic stress and abscisic acid. Plant Mol. Biol., 27:307-315, 1995

Luisa, C., M. Sgherri and F. Navari-Izzo, Sunflower seedlings subjected to increasing water deficit stress: oxidative stress and defence mechanisms. Physiol. Plant 93:25-30, 1995 
Makela, P., K. Jokinen, M. Kontturi, P. Peltonen-Sainio, E. Pheu and S. Somersalo, Foliar application of glycinebetaine a novel product form sugar beet as an approach to increase tomato yield. Ind. Crops Prod., 7:139-148, 1998

Makela, P., P. Peltonen-Sainio, K. Jokinen, E. Pehu, H. Setala, R. Hinkkanen and S. Somessalo,Uptake and traslocation of foliar-applied glycinebetaine in crop plants. Plant Sci., 121:221-230, 1996

Meek, D., D. Oosterhuis and J. gorhuam, Does Foliar applied glycine betaine affect endogenous betaine levels and yield in cotton. Online crop Manage. Doi., 10. 1094/ CM-2003 O 804-02-Rs, 2003

MSTAT Development Team, MSTAT user's guide: A microcomputer program for the design management and analysis of agronomic research experiments. Michigan State Univ. East Lansing, USA, 1989

Naidu, B. P., Simultaneous estimation of sugars, polyols, proline analogues and betaines accumulating in stressed plants by high performance liquid chromatography-Ultra violet detection. Aust. J. Plant Physiol. 25:793-800, 1998

Nandhagopal, A., K. S. Subramanian, A. Gopalan and A. Balasubramanian, Influence of irrigation of critical stress on yield and quality of sunflower. Madras Agric. J., 83: 152-154, 1996

Nolte, K. D., A. D. Hanson and A. D. Gage, Proline accumulation and methylation to proline betaine in citrus :implications for genetic engineering of stress resistance. J. Amer. Soc. Hort. Sci., 122:8-13, 1997
Prabhudeva, T. V., M. M. Chalapathi, S. Thimmegowda, N. Devakhumar, G. G. Rao and K. Mallikarjuna, Soil moisture stress and drought susceptibility index in sunflower. Indian Agri., 42: 287-289, 1998

Rahman, S. Md., H. Miyake and Y. Takeoka, Effects of exogenous glycinebetaine on growth and ultrastructure of salt stressed rice seedlings (Oryza sativa L.). Plant Prod. Sci., 5:33-44, 2002

Rukiye, T. and K. Sebnem, Effects of proline and glycinebetaine on growth and some internal solute changes of cultured tomato embryos under saline conditions. Turk. J. Bot., 17:57-64, 1993

Sakamoto, A. and N. Murata, The role of glycinebetaine in the protection of plants from stress: clues from transgenic plants. Plant Cell Environ., 25:163-171, 2002

Steel, R. G. D. and J. H. Torrie,. Principles and procedures of statistics. McGraw Hill, New York, 1980

Tahir, M. H. N. and S. S. Mehid, Evaluation of open pollinated sunflower (Helianthus annuus L.) populations under water stress and normal conditions. Int. J. Agric. Biolo., 3: 236-23, 2001

Tahir, M. H. N., M. Imran and M. K. Hussain, Evaluation of sunflower (Helianthus annuus L) inbred lines for drought tolerance. Int. J. Agric. Biol., 3: 398-400, 2002

Yancey, P. H., M. E. Clark, S. C. Hand, R. D. Bowels and G. N. Somero, Living with water stress: evolution of osmolyte synthesis. Science., 217:1214-122, 1982 